\title{
Recovering the Unbiased Scene Graphs from the Biased Ones
}

\author{
Meng-Jiun Chiou \\ National University of Singapore \\ mengjiun.chiou@u.nus.edu \\ Changhu Wang \\ ByteDance AI Lab \\ wangchanghu@bytedance.com
}

\author{
Henghui Ding \\ ByteDance AI Lab \\ henghui.ding@bytedance.com \\ Roger Zimmermann \\ National University of Singapore \\ rogerz@comp.nus.edu.sg
}

\author{
Hanshu Yan \\ National University of Singapore \\ hanshu.yan@u.nus.edu \\ Jiashi Feng \\ National University of Singapore \\ elefjia@nus.edu.sg
}

\begin{abstract}
Given input images, scene graph generation (SGG) aims to produce comprehensive, graphical representations describing visual relationships among salient objects. Recently, more efforts have been paid to the long tail problem in SGG; however, the imbalance in the fraction of missing labels of different classes, or reporting bias, exacerbating the long tail is rarely considered and cannot be solved by the existing debiasing methods. In this paper we show that, due to the missing labels, SGG can be viewed as a "Learning from Positive and Unlabeled data" (PU learning) problem, where the reporting bias can be removed by recovering the unbiased probabilities from the biased ones by utilizing label frequencies, i.e., the per-class fraction of labeled, positive examples in all the positive examples. To obtain accurate label frequency estimates, we propose Dynamic Label Frequency Estimation (DLFE) to take advantage of training-time data augmentation and average over multiple training iterations to introduce more valid examples. Extensive experiments show that DLFE is more effective in estimating label frequencies than a naive variant of the traditional estimate, and DLFE significantly alleviates the long tail and achieves state-of-the-art debiasing performance on the VG dataset. We also show qualitatively that SGG models with DLFE produce prominently more balanced and unbiased scene graphs. The source code is publicly available ${ }^{1}$.
\end{abstract}

\section{CCS CONCEPTS}

\section{- Computing methodologies $\rightarrow$ Scene understanding.}

\section{KEYWORDS}

Scene Graph Generation, Reporting Bias, Missing Labels, Long Tail

\section{ACM Reference Format:}

Meng-Jiun Chiou, Henghui Ding, Hanshu Yan, Changhu Wang, Roger Zimmermann, and Jiashi Feng. 2021. Recovering the Unbiased Scene Graphs from the Biased Ones . In Proceedings of the 29th ACM International Conference on Multimedia (MM '21), October 20-24, 2021, Virtual Event, China. ACM, New York, NY, USA, 10 pages. https://doi.org/10.1145/3474085.3475297

${ }^{1}$ https://github.com/coldmanck/recovering-unbiased-scene-graphs

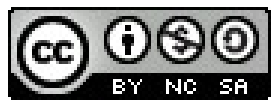

This work is licensed under a Creative Commons Attribution-NonCommercialShareAlike International 4.0 License.

MM '21, October 20-24, 2021, Virtual Event, China. (c) 2021 Copyright held by the owner/author(s).

ACM ISBN 978-1-4503-8651-7/21/10.

https://doi.org/10.1145/3474085.3475297

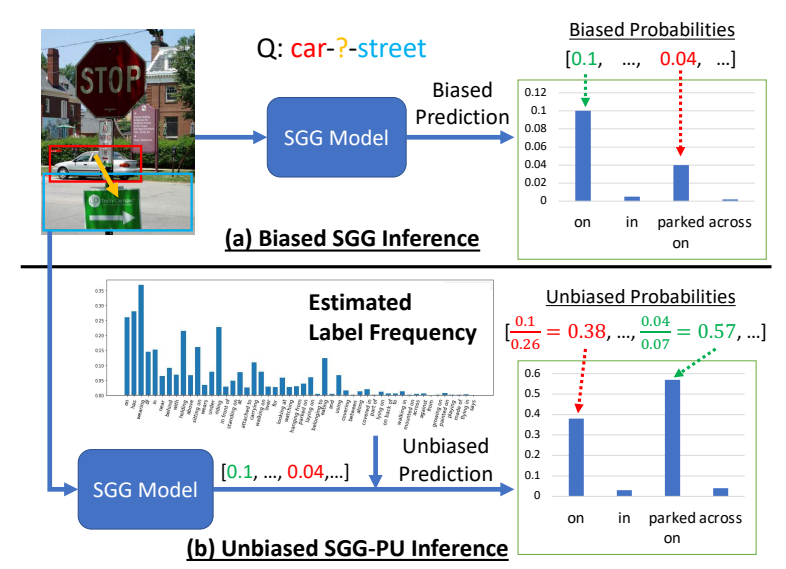

Figure 1: (a) SGG models are usually trained in non-PU setting and output biased probabilities in favor of conspicuous classes. (b) We remove reporting bias by discounting the difference in the chance of being labeled (i.e., label frequency) so that inconspicuous classes are properly predicted.

\section{INTRODUCTION}

Scene graph generation (SGG) [27] aims to predict visual relationships in the form of (subject-predicate-object) among salient objects in images. SGG has been shown to be helpful for image captioning $[21,47,48]$, visual question answering [33, 39], indoor scene understanding $[1,8]$ and thus has been drawing increasing attention $[4-7,9,11,13-15,17-19,25,29,32,37,40-43,45,46,49-53]$.

The long tail problem is common and challenging in SGG [37]: since certain predicates (i.e., head classes) occur far more frequently than others (i.e., tail classes) in the most widely-used VG dataset [20], a model that trained with this unbalanced dataset would favor predicting heads against tails. For instance, the number of training examples of on is $\sim 830 \times$ higher than that of painted on in the VG dataset, and (given ground truth objects) a classical SGG model MOTIFS [53] achieves74.3 Recall@20 for on, in sharp contrast to 0.0 for painted on. However, the fact that the head classes are less descriptive than the tail classes makes the generated scene graphs coarse-grained and less informative, which is not ideal.

Most of the existing efforts in long-tailed SGG [6, 14, 37, 41, $43,45]$ deal with the skewed class distribution directly. However, unlike common long-tailed classification tasks where the long tails are mostly caused by the unbalanced class prior distributions, the long tail of SGG with the VG dataset is significantly affected by the imbalance of missing labels, which remains unsolved. The missing label problem arises as it is unrealistic to annotate the overwhelming 
number of possible visual relations (i.e., $K N(N-1)$ possibilities given $K$ predicate classes and $N$ objects in an image). Training SGG models by treating all unlabeled pairs as negative examples (which is the default setting for most of the existing SGG works) introduces missing label bias in predictions, i.e., predicted probabilities could be under-estimated. What is worse, reporting bias [28, 37] which is prevalent in the VG dataset causes an imbalance in the missing labels of different predicates. That is, the conspicuous classes (e.g., on, in) are more likely to be annotated than the inconspicuous ones (e.g., parked on, covered in). Generally, conspicuous classes are more extensively annotated and have higher label frequencies, i.e., the fraction of labeled, positive examples in all examples of individual classes. The unbalanced label frequency distribution means that the predicted probability of an inconspicuous class could be under-estimated more than that of a conspicuous one, causing a long tail. To produce meaningful scene graphs, the inconspicuous but informative predicates need to be properly predicted. To the best of our knowledge, none of the existing SGG debiasing methods $[6,14,37,41,43,45]$ effectively solve this reporting bias problem.

In this paper, we propose to tackle the reporting bias problem by removing the effect of unbalanced label frequency distribution. That is, we aim to recover the unbiased version of per-class predicted probabilities such that they are independent of the per-class missing label bias. To do this, we first show that learning a SGG model with the VG dataset can viewed as a Learning from Positive and Unlabeled data (PU learning) $[2,10,12]$ problem, where a target PU dataset contains only positive examples and unlabeled data. For clarity, we define that a biased model is trained on a PU dataset by treating the unlabaled data as negatives and outputs biased probabilities, while an unbiased model is trained on a fully-annotated dataset and outputs unbiased probabilities. Under the PU learning setting, the per-class unbiased probabilities are proportional to the biased ones with the per-class label frequencies as the proportionality constants [12]. Motivated by this fact, we propose to recover the unbiased visual relationship probabilities from the biased ones by dividing by the estimated per-class label frequencies so that the imbalance (i.e., reporting bias) can be offset. Especially, the inconspicuous predicates with their probabilities being under-estimated more could then be predicted with higher confidences so that the scene graphs are more informative. An illustrative comparison of the traditional, biased method and our unbiased one is shown in Fig. 1.

A traditional estimator of label frequencies is the per-class average of biased probabilities on a training/validation set predicted by a biased model [12]. While this estimator can work in the easier SGG settings where ground truth bounding boxes are given, i.e., PredCls and SGCls, it is found unable to provide estimates for some classes in the hardest SGG setting where no additional information other than images is provided, i.e. SGDet. The reason is that there are no valid examples (i.e., predicted object pairs that match ground truth boxes and object labels) can be used for label frequency estimation. For instance, by forwarding a trained MOTIFS [53] model on VG training set, 9 out of 50 predicates do not have even a single valid example, making it impossible to estimate. In this paper, we propose to take advantage of the training-time data augmentation such as random flipping to increase the number of valid examples. That is, instead of performing post-training estimations, we propose Dynamic Label Frequency Estimation (DLFE) utilizing augmented training examples by maintaining a moving average of the perbatch biased probability during training. The significant increase in the number of valid examples enables accurate label frequency estimation for unbiased probability recovery.

Our contribution in this work is three-fold. First, we are among the first to tackle the long tail problem in SGG from the perspective of reporting bias, which we remove by recovering the perclass unbiased probability from the biased one with a PU based approach. Second, to obtain accurate label frequency estimates for recovering unbiased probabilities in SGG, we propose DLFE which takes advantage of training-time data augmentation and averages over multiple training iterations to introduce more valid examples. Third, we show that DLFE provides more reliable label frequency estimates than a naive variant of the traditional estimator, and we demonstrate that SGG models with DLFE effectively alleviates the long tail and achieve state-of-the-art debiasing performance with remarkably more informative scene graphs.

\section{RELATED WORK}

\subsection{Scene Graph Generation (SGG)}

SGG [27] aims to generate visual relationships among salient objects. There exists three training and evaluation settings [44, 53]: (1) Predicate Classification (PredCls) predicting relationships given ground truth bounding boxes and object labels, (2) Scene Graph Classification (SGCls) predicting relationships and object labels given bounding boxes and (3) Scene Graph Detection (SGDet) predicting relationships, object labels and bounding boxes with only images.

Typically, SGG models consist of three main modules: proposal generation, object classification, and relationship prediction. Generally, a pre-trained object detection model (e.g., [30]) is adopted for generating proposals. For object classification, instead of using the predictions of object detection models directly, the generated proposals and their features are usually refined into object contexts $[5,38,44,46,53]$ followed by decoding into object labels. A common way to take object contexts into consideration is to run message passing algorithms (e.g., [16, 22, 35]) on a fully-connected $[5,44,46]$, chained [53] or tree-structured [38] graph. For relationship prediction, most approaches $[5,37,53]$ take in object contexts and bounding box features to compute relation contexts with a similar graphical manner. However, not until the recent works $[5,38]$ proposed the less biased mean recall metrics did the research communities in SGG pay attention to the class imbalance problem $[11,13,25,37,41,45]$. Tang et al. [37] borrow the counterfactual idea from causal inference to remove the context-specific bias. Yan et al. [45] propose to perform re-weighting with class relatedness-aware weights. Wang et al. [41] transfer the less-biased knowledge from the secondary learner to the main one with knowledge distillation.

Our proposed method can be viewed as a model-agnostic debiasing method [37, 41, 45]. However, instead of focusing on class relatedness [45], context co-occurrence bias [37] or missing label bias [41], we tackle the underlying reporting bias [28] by dealing with the unbalanced label frequency distribution.

\subsection{Positive Unlabeled (PU) learning}

While the traditional classification setting aims to learn classifiers with both positive and negative data, Learning from Positive and 


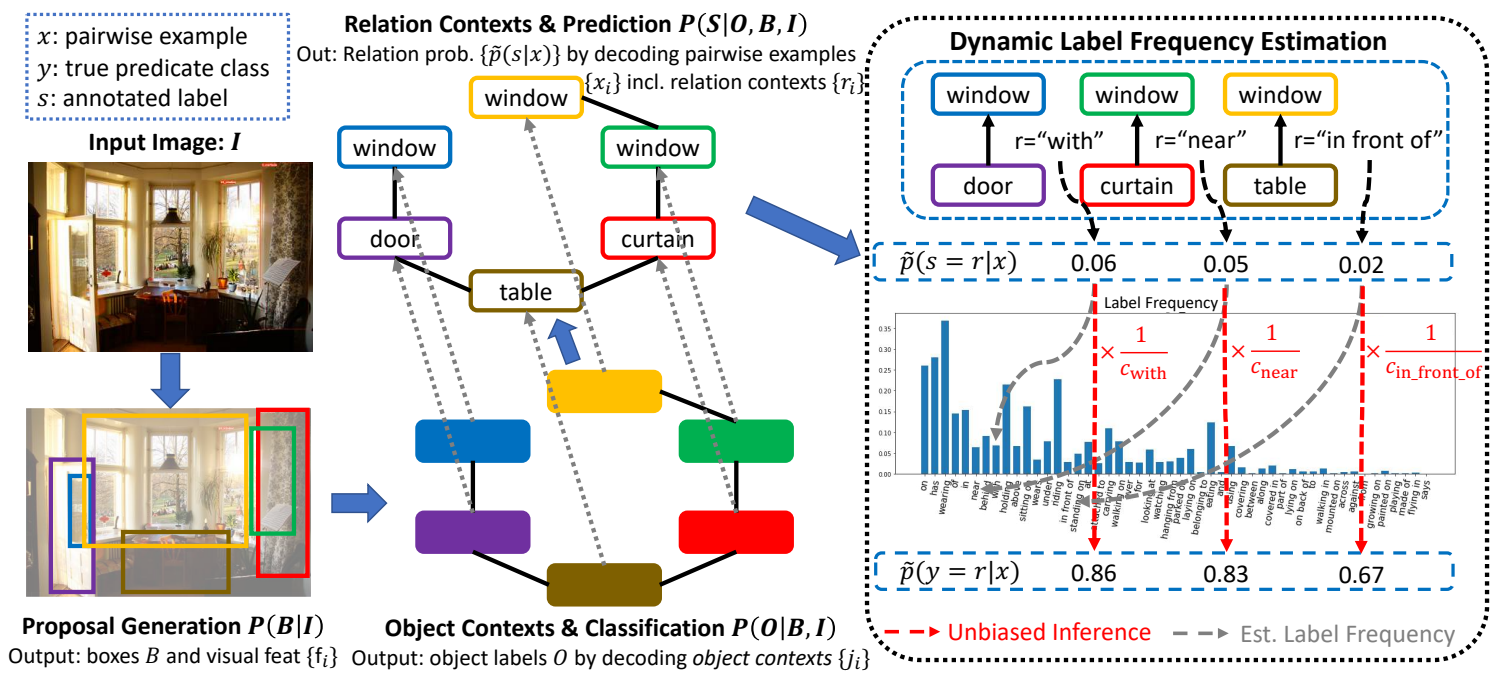

Figure 2: An illustration of training and inferencing a SGG model in a PU manner with Dynamic Label Frequency Estimation (DLFE). Given an input image, proposals and their features are extracted by an object detector. Object classification is performed via message passing on a (e.g., chained [53]) graph followed by object contexts decoding. Object contexts together with bounding boxes and features are then fed into another graph to refine into relation contexts, followed by decoding into the biased probabilities $\tilde{p}(s \mid x)$. DLFE dynamically estimates the label frequencies $c$ with the moving averages of biased probabilities during training. Finally, the unbiased probability of class $r$ is recovered with $\tilde{p}(y=r \mid x)=\frac{1}{c_{r}} \tilde{p}(s=r \mid x)$ during inference.

Unlabeled data, or Positive Unlabeled (PU) learning, is a variant of the traditional setting where a PU dataset contains only positive and unlabeled examples $[2,10,12]$. That is, an unlabeled example can either be truly a negative, or belongs to one or more classes. Learning a biased classifier assuming all unlabeled examples are negative (which is the default setting for most of the existing SGG works) could introduce missing label bias, producing unbalanced predictions. Common PU learning methods can be roughly divided into two categories [2]: (a) training an unbiased model, and (b) inferencing a biased model in a PU manner. We adopt the latter approach in this paper due to its convenience and favorable flexibility.

We note that while Chen et al. [4] also deal with SGG in the PU setting, they do not dive deep into the long tail problem in scene graphs as we do in this paper. They propose a three-stage approach which generates pseudo-labels for the unlabeled examples with a biased trained model, followed by training a less biased model with the additional "positive" examples. However, their approach is time and resource consuming since it requires re-generating pseudo labels if different SGG models are used. Unlike [4], our approach not only can be easily adapted for any SGG model with minimal modification, but is superior in terms of debiasing performance.

\section{METHODOLOGY}

Scene graph generation aims to generate a graph $G$ comprising bounding boxes $B$, object labels $O$, and visual relationships $S$, given an input image $I$. The SGG task $P(G \mid I)$ is usually decomposed into three components for joint training [53]:

$$
P(G \mid I)=P(B \mid I) P(O \mid B, I) P(S \mid O, B, I),
$$

where $P(B \mid I)$ denotes proposal generation, $P(O \mid B, I)$ means object classification and $P(S \mid O, B, I)$ is relationship prediction. We propose to biasedly train a SGG model while we dynamically estimate the label frequencies during training. The estimated label frequencies are then used to recover the unbiased probabilities during inference.

We describe our choice of proposal generation, object classification and relationship prediction in Section 3.1. We then explain how we recover the unbiased probabilities from the biased ones from a PU perspective in Section 3.2, followed by presenting our Dynamic Label Frequency Estimation (DLFE) in Section 3.3. Figure 2 shows an illustration of DLFE applied to SGG models like [38, 53].

\subsection{Model Components}

3.1.1 Proposal Generation $P(B \mid I)$. Given an image $I$, we adopt a pre-trained object detector [30] to extract $N$ object proposals $B=\left\{b_{i} \mid i=1, \ldots, N\right\}$, together with their visual representation $\left\{f_{i} \mid i=1, \ldots, N\right\}$ and $N(N-1)$ union bounding box representations $\left\{f_{i, j} \mid i, j=1, \ldots, N\right\}$ pooled from the output feature map. The visual representations also come with predicted class probabilities: $\left\{p_{i} \mid i=\right.$ $1, \ldots, N\}$ and $\left\{p_{i, j} \mid i, j=1, \ldots, N\right\}$.

3.1.2 Object Classification $P(O \mid B, I)$. For object classification, a graphical representation is constructed which takes in object features $\left\{f_{i}\right\}$ and class probabilities $\left\{p_{i}\right\}$ and outputs object context $\left\{j_{i}\right\}$ refined with message passing algorithms. We experiment our methods with either chained-structured graphs [53] with bi-directional LSTM [16], or tree-structured graphs [38] with TreeLSTM [35]. The output object contexts are then fed into a linear layer followed with a Softmax layer to decode into predicted object labels $O=\left\{o_{i} \mid i=1, \ldots, N\right\}$.

3.1.3 Relationship Prediction $P(S \mid O, B, I)$. Similar to that of object classification, another graphical representation of the same type is established to propagate contexts between features. The module takes in both the object labels $O$ and the object contexts $\left\{j_{i}\right\}$ and outputs refined relation contexts $\left\{r_{i}\right\}$. For each object pair 


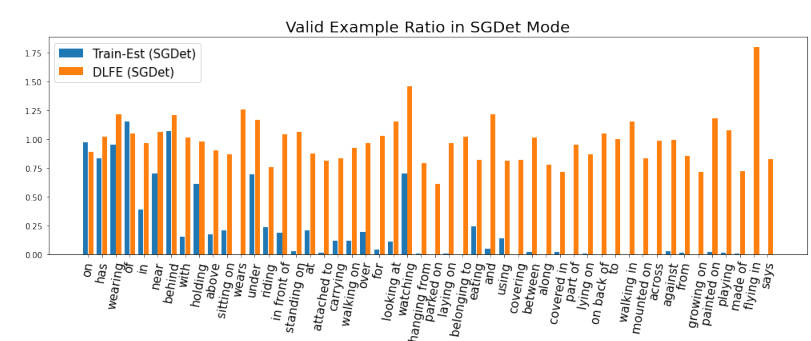

Figure 3: The per-class ratio of valid examples in all examples of VG150 training set [44] (SGDet), by inferencing a trained MOTIFS (Train-Est) or dynamically inferencing a training MOTIFS with augmented data (DLFE).

$\{(i, j) \mid i, j=1, \ldots, N, i \neq j\}$, their relation contexts $\left(r_{i}, r_{j}\right)$, bounding boxes $\left(b_{i}, b_{j}\right)$, union bounding boxes $b_{i j}$ and features $f_{i, j}$ are gathered into a pairwise feature $x_{i j}$ for decoding into a probability vector over the $K$ classes with MLPs followed by a Softmax layer.

\subsection{Recovering the Unbiased Scene Graphs}

Learning SGG from a dataset with missing labels can be viewed as a PU learning problem, which is different from the traditional classification in that (a) no negative examples are available, and (b) unlabeled examples can either be truly negatives or belong to any class. Learning classifiers from a PU dataset by treating all unlabeled data as negatives could introduce strong missing label bias [12], i.e., predicted probabilities could be under-estimated, and reporting bias [28], i.e., predicted probability of an inconspicuous class could be under-estimated more than that of a conspicuous one. We propose to avoid the both biases by recovering the unbiased probabilities, marginalizing the effect of uneven label frequencies.

Given $K$ predicate classes, we denote the visual relation examples taken in by the relationship prediction module of a SGG model by a set of tuple $(x, y, s)$, with $x$ an example (i.e., pairwise object features), $y \in\{0, \ldots, K\}$ the true predicate class ( 0 means the background class) and $s \in\{0, \ldots, K\}$ the relation label (0 means unannotated). The class $y$ cannot be observed from the dataset: while we can derive $y=s$ if the example is labeled $(s \neq 0), y$ can be any number ranging from 0 to $K$ for an unlabeled example $(s=0)$.

For clarity, we now regard $x, y$ and $s$ as random variables. For a target class $r \in\{1, \ldots, K\}$, a biased SGG model is trained to predict the biased probability $P(s=r \mid x)$, which can be derived as follows:

$$
\begin{aligned}
P(s=r \mid x) & =P(s=r, y=r \mid x) \quad \text { (by PU definition) } \\
& =P(y=r \mid x) P(s=r \mid y=r, x),
\end{aligned}
$$

where $P(s=r \mid y=r, x)$ is the probability of example $x$ being selected to be labeled and is called propensity score [2]. Dividing each side by $P(s=r \mid y=r, x)$ we obtain the unbiased probability $P(y=r \mid x)$ :

$$
P(y=r \mid x)=\frac{P(s=r \mid x)}{P(s=r \mid y=r, x)} .
$$

However, as it is unrealistic to obtain the propensity scores of each $x$, the existing works propose to $[4,12]$ bypass the dependence on each $x$ by making the Selected Completely At Random (SCAR) assumption [2]: non-background examples are selected for labeling entirely at random regardless of $x$, i.e., the set of labeled examples is uniformly drawn from the set of positive examples. This means that $P(s=r \mid y=r, x)=P(s=r \mid y=r)$ and Eqn 4 can be written as

$$
P(y=r \mid x)=\frac{P(s=r \mid x)}{P(s=r \mid y=r)},
$$

where $P(s=r \mid y=r)$ is the label frequency of class $r$, or $c_{r}$, which is the fraction of labeled examples in all the examples of class $r$. Notably, discounting the effect of per-class label frequencies in this way also removes the reporting bias. Since label frequencies are usually not provided by annotators, an estimation is required.

\subsection{Dynamic Label Frequency Estimation}

One of the most common estimators of label frequencies, named Train-Est, is the per-class average of biased probabilities $\tilde{p}(s \mid x)$ predicted by a biased model [12] (see full derivation in Appendix 1):

$$
c_{r}=P(s=r \mid y=r) \approx \frac{1}{N_{r}} \sum_{(x, y=r) \in D} \tilde{p}(s=r \mid x),
$$

where $D$ denotes a training or validation set and $N_{r}$ is the cardinality of $\{(x, y=r)\}$. However, we find this way of estimation inconvenient and unsuitable for SGG. To understand why, recall that PredCls, SGCls and SGDet are the three SGG training and evaluation settings, and note that re-estimation of label frequencies is required for each setting since the expected biased probabilities could vary depending on the task difficulty ${ }^{2}$. Firstly, the post-training estimation required before inferencing in each SGG setting is inconvenient and unfavorable. Secondly, the absence of ground truth bounding boxes in SGDet mode results in lack of valid examples for label frequency estimation. For a proposal pair to be valid, its both objects must match ground truth boxes (with IoU $\geq 0.5$ ) and object labels simultaneously. By using Train-Est with MOTIFS [53], as revealed in in Fig. 3 (the blue bars), 9 out of 50 predicates do not have even a valid example, i.e., $\{(x, y=r)\}$ in Eqn. 6 is empty, making it impossible to compute. In addition, more valid examples are missing for inconspicuous classes: as the examples of those classes are concentrated in a much smaller number of images, not matching a bounding box could invalidate lots of examples. A naive remedy is using a default value for those missing estimates; however, as we show in section 4.3 the performance is sub-optimal.

To alleviate this problem, we propose to take advantage of the training-time data augmentation to get back more valid examples for tail classes. Concretely, during training we augment input data by horizontal flipping with a probability of 0.5 , and meanwhile we estimate label frequencies with per-batch biased probabilities. By doing this, the number of valid examples of tail classes could become more normal (and higher) than that of Train-Est, since averaging over augmented examples and multiple training iterations (with varying object label predictions) essentially introduces more samples, which in turn increases the number of valid examples.

Based on this idea, we propose Dynamic Label Frequency Estimation (DLFE) where the main steps are shown in Algorithm 1. In detail, we maintain per-class moving averages of the biased probabilities (Eqn. 6) throughout the training. The estimated label frequencies $\tilde{c}$ are dynamically updated by the per-batch averages $c^{\prime}$ with a momentum $\alpha$ so that the estimates that are more recent matter more: $\tilde{c} \leftarrow \alpha \times c^{\prime}+(1-\alpha) \times \tilde{c}$. Note that for each mini-batch we

\footnotetext{
${ }^{2}$ Using label frequencies estimated in other mode is found to degrade the performance.
} 


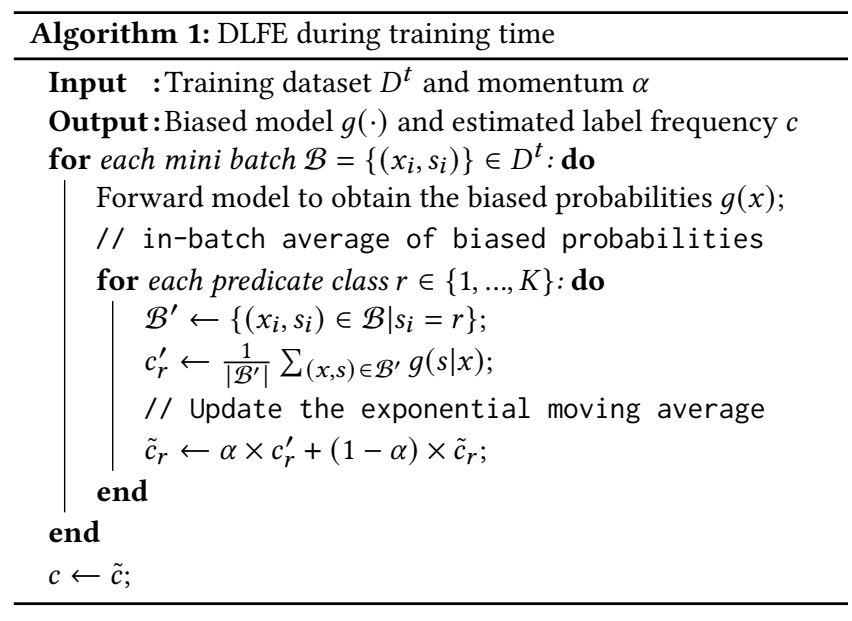

update the estimated $\tilde{c}_{r}$ of class $r$ only if at least one valid example of $r$ presents in the current batch. The estimated values gradually stabilize along with the converging model, and we save the final estimates $c \in \mathbb{R}^{K}$ (as a vector of length $K$ ). During inference, the estimated label frequencies are utilized to recover the unbiased probability distribution $\tilde{p}(y \mid x)$ from the biased one $\tilde{p}(s \mid x)$ by

$$
\tilde{p}(y \mid x)=\frac{1}{c} \odot \tilde{p}(s \mid x),
$$

where $\odot$ denotes the Hadamard (element-wise) product. The average per-epoch number of valid examples obtained by DLFE is shown in Fig. 3 (the tangerine bars), where the inconspicuous classes get remarkably more ( $4 \times$ or more) valid examples. This not only enables accurate estimations for all the classes but makes the estimations easier as no additional, post-training estimation is required.

\section{EXPERIMENTS}

\subsection{Evaluation Settings}

We follow the recent efforts in SGG $[5,53]$ to experiment on a subset of the VG dataset [20] named VG150 [44], which contains 62,723 images for training, 5, 000 for validation and 26, 446 for testing. As discussed earlier, we train and evaluate in the three SGG settings: PredCls, SGCls and SGDet. We evaluate models with, or without graph constraint: whether only a single relation with the highest confidence is predicted for each object pair. Non-graph constraint is denoted as "ng". For evaluation, we adopt recall-based metrics which measures the fraction of ground truth visual relations appearing in top- $K$ confident predictions, where $K$ is 20,50 , or 100 . However, as the plain recall could be dominated by a biased model predicting mostly head classes, we follow [5, 38, 41, 45] to average over per-class recall and focus on the less biased per-class/mean recall (mR@K) and non-graph constraint per-class/mean recall (ng$\mathrm{mR@K})^{3}$. We note that the ng per-class/mean recall should be the fairest measure for debiasing methods since it 1) treats each class equally and 2) reflects the fact that more than one visual relations could exist for an object pair. We follow the long-tailed recognition research [26] to divide the distribution into three parts, including

${ }^{3}$ While results in graph constraint recalls $(\mathrm{R})$ and mean recalls $(\mathrm{mR})$ are less reflective of how unbiased a SGG model is, we provide them for reference in Appendix 4.
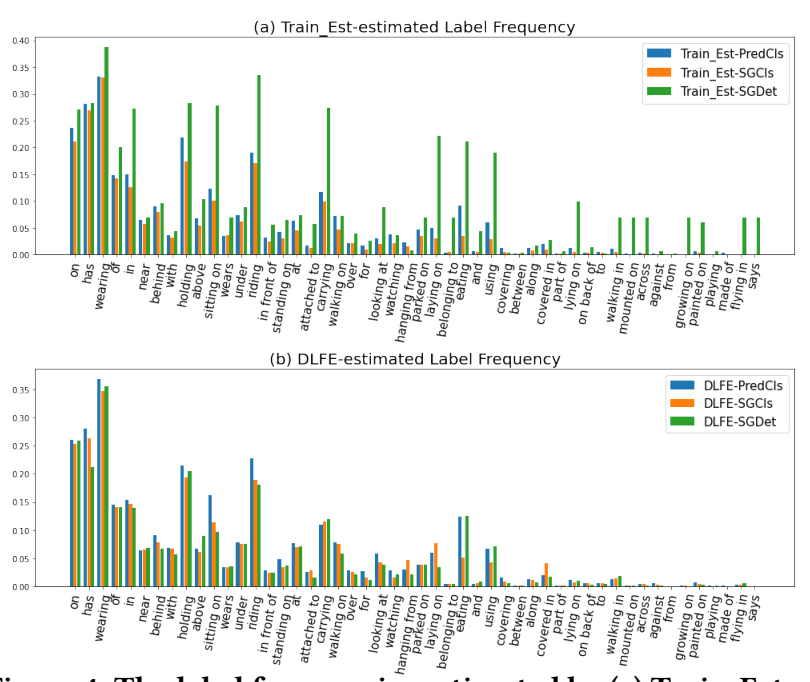

Figure 4: The label frequencies estimated by (a) Train-Est or (b) DLFE with MOTIFS [53]. Predicates sorted by class frequency in descending order.

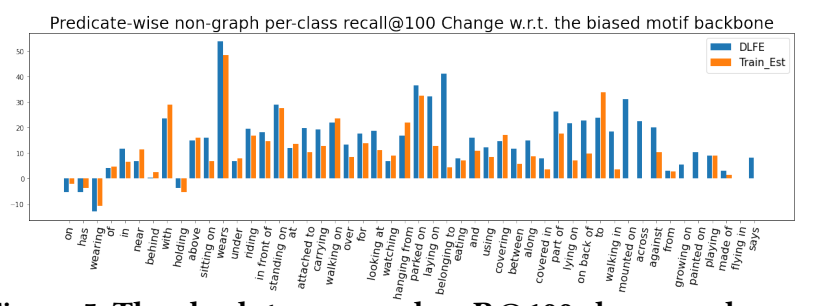

Figure 5: The absolute ng per-class R@100 changes when recovering MOTIFS's [53] unbiased probabilities with the label frequencies estimated by Train-Est or DLFE, in SGDet mode.

head (many-shot; top-15 frequent predicates), middle (medium-shot; mid-20) and tail (few-shot; last-15) and compute their ng-mRs. Note that by DLFE in this section, we mean the dynamic label frequency estimation along with our unbiased scene graph recovery approach.

\subsection{Implementation Details}

As DLFE is a model-agnostic strategy, we experiment with two popular SGG backbones: MOTIFS [53] and VCTree [38]. Following $[37,41]$, we adopt a pre-trained and frozen Faster R-CNN [30] with ResNeXt-101-FPN [23] as the object detector, which achieves 28.14 mAP on VG's testing set [37]. All the hyperparameters, including the momentum $\alpha=0.1$, are tuned with the validation set. All models are trained using SGD optimizer with the initial learning rate of 0.01 after the first 500 iterations of warm-up. Random flipping is applied to all the training examples. The learning rate is decayed, for a maximum of twice, by the factor of 10 once the validation performance plateaus twice consecutively. Training can early stop when the maximum decay step (two) is reached before the maximum 50,000 iterations. The final checkpoint is used for evaluation. The batch size for all experiments is 48 (images). For SGDet setting, we sample 80 proposals from each image and apply per-class NMS [31]. Beside ground truth visual relations, we follow [37] to sample up to 1, 024 pairs with background-to-ground truth ratio being 3:1. 


\begin{tabular}{|c|c|c|c|c|c|c|c|c|c|}
\hline & \multicolumn{3}{|c|}{ Predicate Classification (PredCls) } & \multicolumn{3}{|c|}{ Scene Graph Classification (SGCls) } & \multicolumn{3}{|c|}{ Scene Graph Detection (SGDet) } \\
\hline Model & ng-mR@20 & ng-mR@50 & ng-mR@100 & ng-mR@20 & ng-mR@50 & ng-mR@100 & ng-mR@20 & ng-mR@50 & ng-mR@100 \\
\hline KERN [5] & - & 36.3 & 49.0 & - & 19.8 & 26.2 & - & 11.7 & 16.0 \\
\hline GB-Net- $\beta^{\diamond}[51]$ & - & 44.5 & 58.7 & - & 25.6 & 32.1 & - & 11.7 & 16.6 \\
\hline MOTIFS $^{\dagger}[41,53]$ & 19.9 & 32.8 & 44.7 & 11.3 & 19.0 & 25.0 & 7.5 & 12.5 & 16.9 \\
\hline MOTIFS-Reweight ${ }^{\ddagger}$ & 20.5 & 33.5 & 44.4 & 12.6 & 19.1 & 24.3 & 8.0 & 12.9 & 16.8 \\
\hline MOTIFS-L2+uKD ${ }^{\ddagger}[41]$ & - & 36.9 & 50.9 & - & 22.7 & 30.1 & - & 14.0 & 19.5 \\
\hline MOTIFS-L2+cKD ${ }^{\ddagger}[41]$ & - & 37.2 & 50.8 & - & 22.1 & 29.6 & - & 14.2 & 19.8 \\
\hline MOTIFS-TDE $^{\dagger}[37]$ & 18.7 & 29.0 & 38.2 & 10.7 & 16.1 & 21.1 & 7.4 & 11.2 & 14.9 \\
\hline MOTIFS-PCPL ${ }^{\dagger}[45]$ & 25.6 & 38.5 & 49.3 & 13.1 & 19.9 & 25.6 & 9.8 & 14.8 & 19.6 \\
\hline MOTIFS-STL $^{\dagger}[4]$ & 15.7 & 29.4 & 43.2 & 10.3 & 18.4 & 27.2 & 6.4 & 10.6 & 15.0 \\
\hline MOTIFS-DLFE & 30.0 & 45.8 & 57.7 & 17.6 & 25.6 & 32.0 & 11.7 & 18.1 & 23.0 \\
\hline VCTree $^{\dagger}[38,41]$ & 21.4 & 35.6 & 47.8 & 14.3 & 23.3 & 31.4 & 7.5 & 12.5 & 16.7 \\
\hline VCTree-Reweight & 20.6 & 32.5 & 41.6 & 14.1 & 21.3 & 27.8 & 8.0 & 12.1 & 15.9 \\
\hline VCTree-L2+uKD ${ }^{\ddagger}$ [41] & - & 37.7 & 51.7 & - & 26.8 & 35.2 & - & 13.8 & 19.1 \\
\hline VCTree-L2+cKD $\$$ [41] & - & 38.4 & 52.4 & - & 26.8 & 35.8 & - & 13.9 & 19.0 \\
\hline VCTree-TDE $^{\dagger}[37]$ & 20.9 & 32.4 & 41.5 & 12.4 & 19.1 & 25.5 & 7.8 & 11.5 & 15.2 \\
\hline VCTree-PCPL ${ }^{\dagger}[45]$ & 25.1 & 38.5 & 49.3 & 17.2 & 25.9 & 32.7 & 9.9 & 15.1 & 19.9 \\
\hline VCTree-STL $^{\dagger}[4]$ & 16.8 & 31.8 & 45.1 & 12.7 & 22.0 & 32.7 & 6.0 & 10.0 & 14.1 \\
\hline VCTree-DLFE & 29.1 & 44.6 & 56.8 & 21.6 & 31.4 & 38.8 & 11.7 & 17.5 & 22.5 \\
\hline
\end{tabular}

Table 1: Performance comparison in ng-mR@K on VG150 [20, 44]. Models in the first section are with VGG16 backbone [34]. $\dagger$ models implemented or reproduced ourselves with ResNeXt-101-FPN [23] backbone. $\ddagger$ models also with the same ResNeXt101-FPN backbone while their performance are reported by the respective papers. ${ }^{\diamond}$ model using external knowledge bases.

\begin{tabular}{|c|c|c|c|c|c|c|c|c|c|}
\hline & \multicolumn{3}{|c|}{ Predicate Classification (PredCls) } & \multicolumn{3}{|c|}{ Scene Graph Classification (SGCls) } & \multicolumn{3}{|c|}{ Scene Graph Detection (SGDet) } \\
\hline Model & mR@20 & mR@50 & mR@100 & mR@20 & mR@50 & mR@100 & mR@20 & mR@50 & mR@100 \\
\hline $\mathrm{IMP}+[5,44]$ & - & 9.8 & 10.5 & - & 5.8 & 6.0 & - & 3.8 & 4.8 \\
\hline FREQ $[38,53]$ & 8.3 & 13.0 & 16.0 & 5.1 & 7.2 & 8.5 & 4.5 & 6.1 & 7.1 \\
\hline MOTIFS $[38,53]$ & 10.8 & 14.0 & 15.3 & 6.3 & 7.7 & 8.2 & 4.2 & 5.7 & 6.6 \\
\hline KERN [5] & - & 17.7 & 19.2 & - & 9.4 & 10.0 & - & 6.4 & 7.3 \\
\hline VCTree [38] & 14.0 & 17.9 & 19.4 & 8.2 & 10.1 & 10.8 & 5.2 & 6.9 & 8.0 \\
\hline GPS-Net [25] & 17.4 & 21.3 & 22.8 & 10.0 & 11.8 & 12.6 & 6.9 & 8.7 & 9.8 \\
\hline GB-Net- $\beta^{\diamond}[51]$ & - & 22.1 & 24.0 & - & 12.7 & 13.4 & - & 7.1 & 8.5 \\
\hline MOTIFS $^{\dagger}[37,53]$ & 13.0 & 16.5 & 17.8 & 7.2 & 8.9 & 9.4 & 5.3 & 7.3 & 8.6 \\
\hline MOTIFS-Focal ${ }^{\ddagger}[24,37]$ & 10.9 & 13.9 & 15.0 & 6.3 & 7.7 & 8.3 & 3.9 & 5.3 & 6.6 \\
\hline MOTIFS-Resample ${ }^{\ddagger}[3,37]$ & 14.7 & 18.5 & 20.0 & 9.1 & 11.0 & 11.8 & 5.9 & 8.2 & 9.7 \\
\hline MOTIFS-Reweight ${ }^{\dagger}$ & 14.3 & 17.3 & 18.6 & 9.5 & 11.2 & 11.7 & 6.7 & 9.2 & 10.9 \\
\hline MOTIFS-L2+uKD ${ }^{\ddagger}[41]$ & 14.2 & 18.6 & 20.3 & 8.6 & 10.9 & 11.8 & 5.7 & 7.9 & 9.5 \\
\hline MOTIFS-L2+cKD ${ }^{+}[41]$ & 14.4 & 18.5 & 20.2 & 8.7 & 10.7 & 11.4 & 5.8 & 8.1 & 9.6 \\
\hline MOTIFS-TDE $^{\dagger}[37]$ & 17.4 & 24.2 & 27.9 & 9.9 & 13.1 & 14.9 & 6.7 & 9.2 & 11.1 \\
\hline MOTIFS-PCPL ${ }^{\dagger}[45]$ & 19.3 & 24.3 & 26.1 & 9.9 & 12.0 & 12.7 & 8.0 & 10.7 & 12.6 \\
\hline MOTIFS-STL ${ }^{\dagger}[4]$ & 13.3 & 20.1 & 22.3 & 8.5 & 12.8 & 14.1 & 5.4 & 7.6 & 9.1 \\
\hline MOTIFS-DLFE & 22.1 & 26.9 & 28.8 & 12.8 & 15.2 & 15.9 & 8.6 & 11.7 & 13.8 \\
\hline VCTree $^{\dagger}[37,38]$ & 14.1 & 17.7 & 19.1 & 9.1 & 11.3 & 12.0 & 5.2 & 7.1 & 8.3 \\
\hline VCTree-Reweight $^{\dagger}$ & 16.3 & 19.4 & 20.4 & 10.6 & 12.5 & 13.1 & 6.6 & 8.7 & 10.1 \\
\hline VCTree-L2+uKD ${ }^{\ddagger}[41]$ & 14.2 & 18.2 & 19.9 & 9.9 & 12.4 & 13.4 & 5.7 & 7.7 & 9.2 \\
\hline VCTree-L2+cKD ${ }^{+}[41]$ & 14.4 & 18.4 & 20.0 & 9.7 & 12.4 & 13.1 & 5.7 & 7.7 & 9.1 \\
\hline VCTree-TDE $^{\dagger}[37]$ & 19.2 & 26.2 & 29.6 & 11.2 & 15.2 & 17.5 & 6.8 & 9.5 & 11.4 \\
\hline VCTree-PCPL $^{\dagger}[45]$ & 18.7 & 22.8 & 24.5 & 12.7 & 15.2 & 16.1 & 8.1 & 10.8 & 12.6 \\
\hline VCTree-STL $^{\dagger}[4]$ & 14.3 & 21.4 & 23.5 & 10.5 & 14.6 & 16.6 & 5.1 & 7.1 & 8.4 \\
\hline VCTree-DLFE & 20.8 & 25.3 & 27.1 & 15.8 & 18.9 & 20.0 & 8.6 & 11.8 & 13.8 \\
\hline
\end{tabular}

Table 2: Performance comparison of SGG models in graph-constraint mR@ $K$ on VG150 [20, 44] testing set. Models in the first section are with VGG16 while the others are with ResNeXt-101-FPN. $\dagger, \ddagger$ and $\diamond$ are with the same meanings as in Table 1 .

\subsection{Comparing DLFE to Train-Est}

We aim to answer the question: whether DLFE is more effective in estimating label frequency than Train-Est, by comparing 1) the consistencies of the estimated label frequencies and 2) their debiasing performance. As discussed earlier that label frequencies of the predicates lacking a valid example cannot be estimated by Train-Est, we thus naively assign the median of the other estimated label frequencies to those missing values.

A comparison of the estimated label frequencies is presented in Fig. 4. It is clear from (a) that, even for the classes that with at least one valid example, the Train-Est estimated values tend to be abnormally high in SGDet setting. Note that while there might be differences in estimated values for different SGG settings, with a same backbone they should still be relatively similar. In contrast, (b) shows DLFE-estimated values are more consistent across the three settings. We also compare their debiasing performance in SGDet ${ }^{4}$ with the absolute ng per-class R@100 change in Fig. 5. Apparently, Train-Est barely improves the per-class recalls especially for tail

\footnotetext{
${ }^{4}$ Results in numbers and the other two SGG settings are provided in Appendix 2.
} 


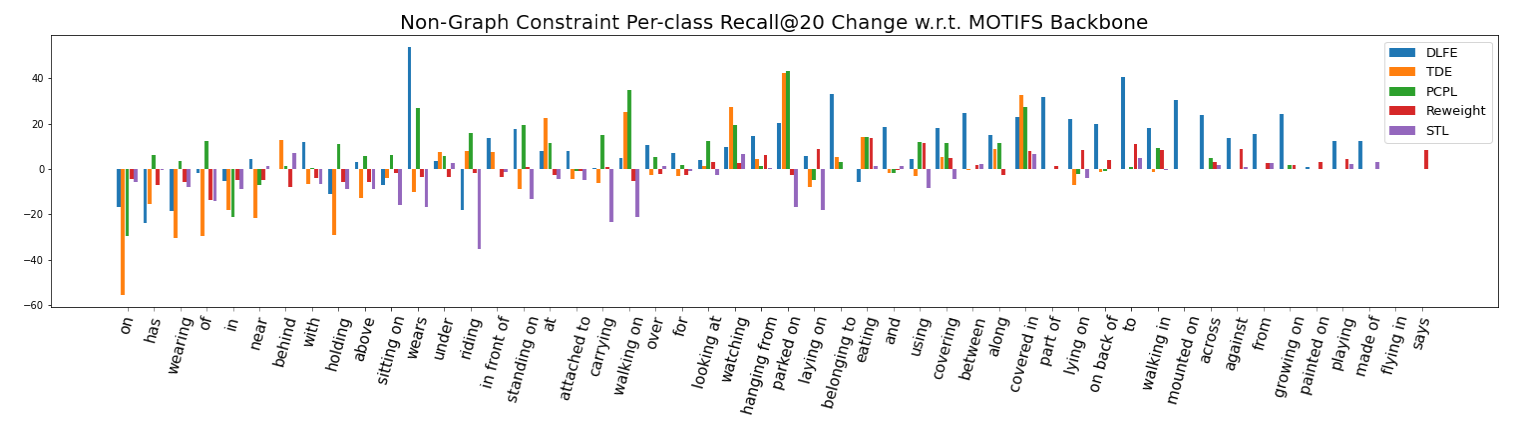

Figure 6: Non-graph constraint per-class Recall@20 (PredCls) change w.r.t. MOTIFS baseline. DLFE significantly improves the mid-to-tail recalls (where the other debiasing methods struggle) without compromising much head classes performance.

\begin{tabular}{l|cc|cc|cc}
\hline \multicolumn{3}{c|}{ Head Recalls } & \multicolumn{2}{c}{ Middle Recalls } & \multicolumn{2}{c}{ Tail Recalls } \\
\hline Model & R@50 & R@100 & R@50 & R@100 & R@50 & R@100 \\
\hline MOTIFS $^{\dagger}[37,53]$ & 65.9 & $\mathbf{7 8 . 6}$ & 30.0 & 45.4 & 3.3 & 9.7 \\
MOTIFS-Reweight $^{\dagger}$ & 57.4 & 69.2 & 30.7 & 43.0 & 13.3 & 21.5 \\
MOTIFS-TDE $^{\dagger}[37]$ & 48.3 & 60.8 & 34.9 & 46.1 & 1.8 & 5.3 \\
MOTIFS-PCPL $^{\dagger}[45]$ & $\mathbf{6 6 . 5}$ & 77.6 & 41.8 & $\mathbf{5 5 . 2}$ & 6.0 & 13.2 \\
MOTIFS-STL $^{\dagger}[4]$ & 56.4 & 70.0 & 24.1 & 39.8 & 9.6 & 21.2 \\
MOTIFS-DLFE & 61.9 & 72.4 & $\mathbf{4 2 . 8}$ & 54.2 & $\mathbf{3 1 . 8}$ & $\mathbf{4 4 . 6}$ \\
\hline VCTree $^{\dagger}[37,38]$ & $\mathbf{6 7 . 5}$ & $\mathbf{7 9 . 8}$ & 34.3 & 50.0 & 5.5 & 12.7 \\
VCTree-Reweight $^{\dagger}$ & 61.6 & 73.4 & 28.3 & 38.3 & 9.0 & 14.3 \\
VCTree-TDE $^{\dagger}[37]$ & 54.8 & 67.5 & 37.9 & 49.1 & 2.5 & 5.4 \\
VCTree-PCPL $^{\dagger}[45]$ & 64.5 & 75.9 & $\mathbf{4 2 . 6}$ & $\mathbf{5 4 . 2}$ & 6.9 & 16.1 \\
VCTree-STL $^{\dagger}[4]$ & 57.6 & 71.1 & 26.1 & 41.8 & 13.8 & 23.5 \\
VCTree-DLFE & 57.5 & 68.3 & 36.0 & 48.2 & $\mathbf{2 6 . 5}$ & $\mathbf{3 8 . 1}$ \\
\hline
\end{tabular}

Table 3: Non-graph constraint head, middle and tail recalls (PredCls). $\dagger$ is with the same meaning as in Table 1. DLFE improves the tail recalls remarkably and achieves new SOTA.

classes that lacks enough valid examples, while DLFE achieves higher and more consistent improvement across all the predicates.

These results verify the claim that, apart from being more convenient (requiring no post-training estimation), DLFE is more effective than naive Train-Est for providing more reliable estimates.

\subsection{Comparing to other Debiasing Methods}

While we list the results of different SGG backbone model for reference, we mainly compare our approach with the model-agnostic debiasing methods including Focal Loss [24], Resampling [3], Reweighting, $L 2+\{u, c\} K D$ [41], TDE [37], PCPL [45] and STL [4]. L2+\{u,c\}KD is a two-learner knowledge distillation framework for reducing dataset biases. TDE is an inference-time debiasing method which ensembles counterfactual thinking by removing context-specific bias. PCPL learns the relatedness scores among predicates which are used as the weights in reweighting, and is the current state-of-theart in terms of mR. STL generates soft pseudo-labels for unlabeled data used for joint training. We re-implement PCPL and STL since their backbone is not directly comparable, and Reweighting since there does not exist its reported performance for VCTree. We report our reproduced results of TDE with the authors' codebase [36].

The results in ng-mR@K are presented in Table 1, where DLFE significantly improves ng-mRs for both MOTIFS and VCTree and outperforms the existing debiasing methods. Notably, TDE, which was proposed to alleviate the long tail by removing the contextspecific bias, is shown to adversely affect the ability of predicting

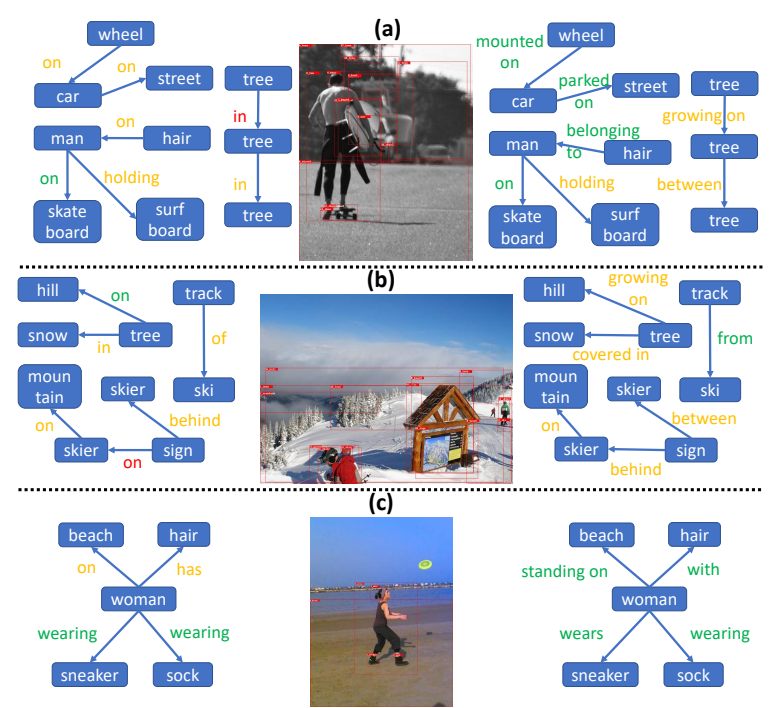

Figure 7: Scene graphs generated by MOTIFS (left) and MOTIFS-DLFE (right) in PredCls. Only the top-1 prediction is shown for each object pair. A prediction can be correct (matches GT), incorrect (does not match GT and weird) or acceptable (does not match GT but still reasonable).

multiple relations for per object pair. This shows that removing the reporting bias is more beneficial for debiasing SGG models.

While the graph-constraint $\mathrm{mR}$ metric does not reflect the fact that multiple relations could exist between an object pair, due to its popularity we still present the results in Table 2. Debiasing MOTIFS with our proposed DLFE still significantly improves mean recalls, achieving state-of-the-art $\mathrm{mR}$ across all the three settings. Large performance boost are also seen in VCTree with DLFE, and new SOTAs are attained for PredCls (mR@20), SGCls and SGDet.

To better understand how DLFE affects the performance of each class, we also present non-graph constraint per-class recall@20 changes compared to MOTIFS backbone-only (biased classifier), in Figure 6. While all the debiasing methods increase the recall of the less frequent, middle-to-tail classes, only DLFE improves the tail (last-15) classes' performance significantly. The other approach that also visibly improves the tail classes' performance is Reweighting; however, their relatively small improvements demonstrate that naively dealing with the unbalanced class frequencies is less effective than tackling the reporting bias. 


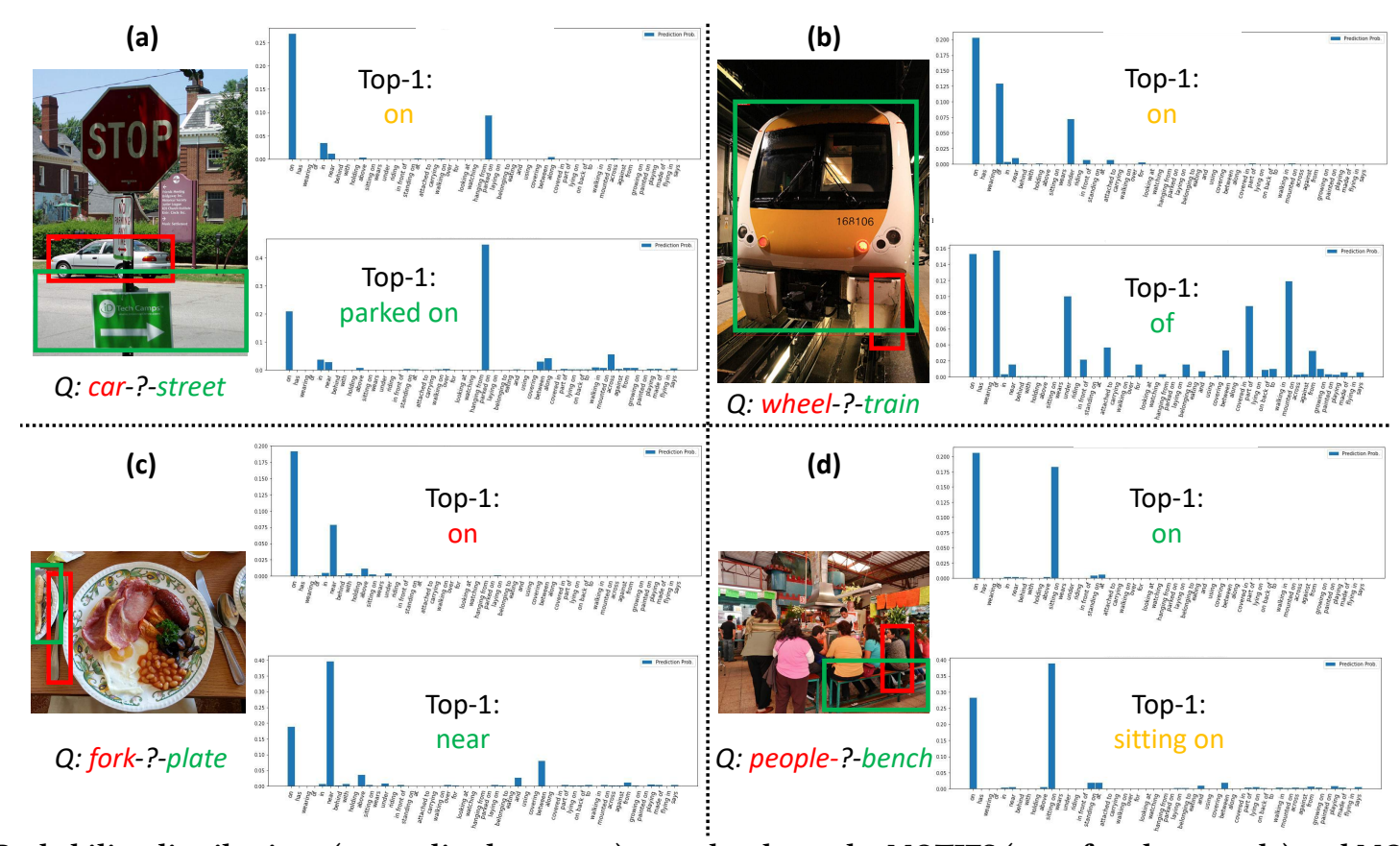

Figure 8: Probability distributions (normalized to sum 1) over the classes by MOTIFS (top of each example) and MOTIFS-DLFE (bottom). The top-1 predictions can be correct (GT), incorrect (Non-GT and weird) or acceptable (Non-GT but reasonable).

We also present the head (many shot), middle (medium shot) and tail (few shot) non-graph constraint recalls in PredCls ${ }^{5}$ with the MOTIFS backbone in Table 3. Remarkably, DLFE outperforms the others by significant margins regarding the tail recalls, e.g., Tail R@50 is 31.8 for DLFE, versus 1.8/13.3 for TDE/Reweighting, showing that DLFE is especially good at dealing with the long tail.

\subsection{Qualitative Results}

The scene graphs of three testing images are visualized in Figure 7, where the scene graphs on the left side are generated by MOTIFS and those on the right are by MOTIFS-DLFE. (a) is an apparent example that, while wheel-on-car, car-on-street, hair-onman predicted by MOTIFS are reasonable, wheel-mounted oncar,car-parked on-street,hair-belonging to-man predicted by MOTIFS-DLFE match the ground truth and are also more descriptive (while being inconspicuous). Similarly, treegrowing on-hill in Example(b) and woman-standing onbeach in (c) are also correct and more descriptive; however, due to the missing label issue in the VG dataset, tree-growing onhill can not be correctly recalled (shown as tangerine color).

To understand how DLFE changes the probability distribution, we visualize the biased (MOTIFS) and unbiased (MOTIFS-DLFE) probabilities, given a subject-object pair, in Figure $8 .{ }^{6}$ Prediction confidences are shown to be calibrated towards minor but expressive predicates like (a) car-parked on-street, (b) wheelof-train,(d)people-sitting on-bench(while sitting on is not in the ground truth). Notably in (c), fork is actually not on the plate but was mis-predicted by MOTIFS due to the strong bias (i.e., many fork-in/on-plate examples in VG dataset),

\footnotetext{
${ }^{5}$ The full results including graph-constraint, SGCls/SGDet are available in Appendix 3. ${ }^{6}$ More visualizations are available in Appendix 5.
}

while MOTIFS-DLFE correctly predicts near. Moreover, (b) shows that the confidences of MOTIFS-DLFE for predicates other than the GT of, such as mounted on and part of, have increased remarkably, presumably because they are also reasonable choices. This demonstrates the effectiveness of DLFE for balanced SGG.

\section{CONCLUSIONS}

In this paper, we deal with the long tail problem in SGG with the cause (unbalanced missing labels) instead of its superficial effect (long-tailed class distribution). To ward off the reporting bias caused by the imbalance in missing labels, we view SGG as a PU learning problem and we remove the per-class missing label bias by recovering the unbiased probabilities from the biased ones. To obtain reliable label frequencies for unbiased probability recovery, we take advantage of the data augmentation during training and perform Dynamic Label Frequency Estimation (DLFE) which maintains the moving averages of per-class biased probability and effectively introduces more valid samples, especially in SGDet training and evaluation mode. Extensive quantitative and qualitative experiments demonstrate that DLFE is more effective in estimating label frequencies than a naive variant of a traditional estimator, and SGG models with DLFE achieve state-of-the-art debiasing performance on the VG dataset, producing well-balanced scene graphs.

\section{ACKNOWLEDGMENTS}

This research is supported by Singapore Ministry of Education Academic Research Fund Tier 1 under MOE's official grant number T1 251RES2029, Singapore National Research Foundation ("CogniVision" grant NRF-CRP20-2017-0003) and its AI Singapore Programme (AISG Award No: AISG-100E-2019-035) and Medium Sized Center for Advanced Robotics Technology Innovation. 


\section{REFERENCES}

[1] Iro Armeni, Zhi-Yang He, JunYoung Gwak, Amir R Zamir, Martin Fischer, Jitendra Malik, and Silvio Savarese. 2019. 3d scene graph: A structure for unified semantics, $3 \mathrm{~d}$ space, and camera. In Proceedings of the IEEE/CVF International Conference on Computer Vision. 5664-5673.

[2] Jessa Bekker and Jesse Davis. 2020. Learning from positive and unlabeled data: A survey. Machine Learning 109, 4 (2020), 719-760.

[3] Evgeny Burnaev, Pavel Erofeev, and Artem Papanov. 2015. Influence of resampling on accuracy of imbalanced classification. In Eighth international conference on machine vision (ICMV 2015), Vol. 9875. International Society for Optics and Photonics, 987521.

[4] Diqi Chen, Xiaodan Liang, Yizhou Wang, and Wen Gao. 2019. Soft Transfer Learning via Gradient Diagnosis for Visual Relationship Detection. In 2019 IEEE Winter Conference on Applications of Computer Vision (WACV). IEEE, 1118-1126.

[5] Tianshui Chen, Weihao Yu, Riquan Chen, and Liang Lin. 2019. Knowledgeembedded routing network for scene graph generation. In Proceedings of the IEEE Conference on Computer Vision and Pattern Recognition. 6163-6171.

[6] Vincent S Chen, Paroma Varma, Ranjay Krishna, Michael Bernstein, Christopher Re, and Li Fei-Fei. 2019. Scene graph prediction with limited labels. In Proceedings of the IEEE/CVF International Conference on Computer Vision. 2580-2590.

[7] Meng-Jiun Chiou, Chun-Yu Liao, Li-Wei Wang, Roger Zimmermann, and Jiashi Feng. 2021. ST-HOI: A Spatial-Temporal Baseline for Human-Object Interaction Detection in Videos. arXiv preprint arXiv:2105.11731 (2021).

[8] Meng-Jiun Chiou, Zhenguang Liu, Yifang Yin, An-An Liu, and Roger Zimmermann. 2020. Zero-Shot Multi-View Indoor Localization via Graph Location Networks. In Proceedings of the 28th ACM International Conference on Multimedia. 3431-3440.

[9] Meng-Jiun Chiou, Roger Zimmermann, and Jiashi Feng. 2021. Visual Relationship Detection With Visual-Linguistic Knowledge From Multimodal Representations. IEEE Access 9 (2021), 50441-50451.

[10] François Denis, Rémi Gilleron, and Fabien Letouzey. 2005. Learning from positive and unlabeled examples. Theoretical Computer Science 348, 1 (2005), 70-83.

[11] Apoorva Dornadula, Austin Narcomey, Ranjay Krishna, Michael Bernstein, and Fei-Fei Li. 2019. Visual relationships as functions: Enabling few-shot scene graph prediction. In Proceedings of the IEEE/CVF International Conference on Computer Vision Workshops. 0-0.

[12] Charles Elkan and Keith Noto. 2008. Learning classifiers from only positive and unlabeled data. In Proceedings of the 14th ACM SIGKDD international conference on Knowledge discovery and data mining. 213-220.

[13] Jiuxiang Gu, Handong Zhao, Zhe Lin, Sheng Li, Jianfei Cai, and Mingyang Ling. 2019. Scene graph generation with external knowledge and image reconstruction. In Proceedings of the IEEE Conference on Computer Vision and Pattern Recognition. 1969-1978.

[14] Tao He, Lianli Gao, Jingkuan Song, Jianfei Cai, and Yuan-Fang Li. 2020. Learning from the Scene and Borrowing from the Rich: Tackling the Long Tail in Scene Graph Generation. In Proceedings of the Twenty-Ninth International foint Conference on Artificial Intelligence, IfCAI-20. 587-593. Main track.

[15] Roei Herzig, Moshiko Raboh, Gal Chechik, Jonathan Berant, and Amir Globerson 2018. Mapping images to scene graphs with permutation-invariant structured prediction. In Advances in Neural Information Processing Systems. 7211-7221.

[16] Sepp Hochreiter and Jürgen Schmidhuber. 1997. Long short-term memory. Neural computation 9, 8 (1997), 1735-1780.

[17] Zih-Siou Hung, Arun Mallya, and Svetlana Lazebnik. 2020. Contextual translation embedding for visual relationship detection and scene graph generation. IEEE transactions on pattern analysis and machine intelligence (2020).

[18] Mahmoud Khademi and Oliver Schulte. 2020. Deep Generative Probabilistic Graph Neural Networks for Scene Graph Generation. Proceedings of the AAAI Conference on Artificial Intelligence 34, 07 (Apr. 2020), 11237-11245. https: //doi.org/10.1609/aaai.v34i07.6783

[19] Boris Knyazev, Harm de Vries, Cătălina Cangea, Graham W Taylor, Aaron Courville, and Eugene Belilovsky. 2020. Graph Density-Aware Losses for Novel Compositions in Scene Graph Generation. In British Machine Vision Conference (BMVC).

[20] Ranjay Krishna, Yuke Zhu, Oliver Groth, Justin Johnson, Kenji Hata, Joshua Kravitz, Stephanie Chen, Yannis Kalantidis, Li-Jia Li, David A Shamma, et al. 2017. Visual genome: Connecting language and vision using crowdsourced dense image annotations. International fournal of Computer Vision 123, 1 (2017), 32-73.

[21] Xiangyang Li and Shuqiang Jiang. 2019. Know more say less: Image captioning based on scene graphs. IEEE Transactions on Multimedia 21, 8 (2019), 2117-2130.

[22] Yujia Li, Daniel Tarlow, Marc Brockschmidt, and Richard Zemel. 2015. Gated graph sequence neural networks. arXiv preprint arXiv:1511.05493 (2015)

[23] Tsung-Yi Lin, Piotr Dollár, Ross Girshick, Kaiming He, Bharath Hariharan, and Serge Belongie. 2017. Feature pyramid networks for object detection. In Proceedings of the IEEE conference on computer vision and pattern recognition. 2117-2125.

[24] Tsung-Yi Lin, Priya Goyal, Ross Girshick, Kaiming He, and Piotr Dollár. 2017. Focal loss for dense object detection. In Proceedings of the IEEE international conference on computer vision. 2980-2988.
[25] Xin Lin, Changxing Ding, Jinquan Zeng, and Dacheng Tao. 2020. GPS-Net: Graph Property Sensing Network for Scene Graph Generation. In Proceedings of the IEEE/CVF Conference on Computer Vision and Pattern Recognition (CVPR).

[26] Ziwei Liu, Zhongqi Miao, Xiaohang Zhan, Jiayun Wang, Boqing Gong, and Stella X Yu. 2019. Large-scale long-tailed recognition in an open world. In Proceedings of the IEEE/CVF Conference on Computer Vision and Pattern Recognition. 2537-2546.

[27] Cewu Lu, Ranjay Krishna, Michael Bernstein, and Li Fei-Fei. 2016. Visual relationship detection with language priors. In European Conference on Computer Vision. Springer, 852-869.

[28] Ishan Misra, C Lawrence Zitnick, Margaret Mitchell, and Ross Girshick. 2016. Seeing through the human reporting bias: Visual classifiers from noisy humancentric labels. In Proceedings of the IEEE Conference on Computer Vision and Pattern Recognition. 2930-2939.

[29] G. Ren, L. Ren, Y. Liao, S. Liu, B. Li, J. Han, and S. Yan. 2021. Scene Graph Generation With Hierarchical Context. IEEE Transactions on Neural Networks and Learning Systems 32, 2 (2021), 909-915. https://doi.org/10.1109/TNNLS.2020. 2979270

[30] Shaoqing Ren, Kaiming He, Ross Girshick, and Jian Sun. 2015. Faster r-cnn: Towards real-time object detection with region proposal networks. In Advances in neural information processing systems. 91-99.

[31] Azriel Rosenfeld and Mark Thurston. 1971. Edge and curve detection for visual scene analysis. IEEE Transactions on computers 100, 5 (1971), 562-569.

[32] Sahand Sharifzadeh, Sina Moayed Baharlou, and Volker Tresp. 2020. Classification by Attention: Scene Graph Classification with Prior Knowledge. arXiv preprint arXiv:2011.10084 (2020).

[33] Jiaxin Shi, Hanwang Zhang, and Juanzi Li. 2019. Explainable and explicit visual reasoning over scene graphs. In Proceedings of the IEEE Conference on Computer Vision and Pattern Recognition. 8376-8384.

[34] Karen Simonyan and Andrew Zisserman. 2014. Very deep convolutional networks for large-scale image recognition. arXiv preprint arXiv:1409.1556 (2014).

[35] Kai Sheng Tai, Richard Socher, and Christopher D. Manning. 2015. Improved Semantic Representations From Tree-Structured Long Short-Term Memory Networks. In Proceedings of the 53rd Annual Meeting of the Association for Computational Linguistics and the 7th International foint Conference on Natural Language Processing. Association for Computational Linguistics, Beijing, China, 1556-1566.

[36] Kaihua Tang. 2020. A Scene Graph Generation Codebase in PyTorch. https: //github.com/KaihuaTang/Scene-Graph-Benchmark.pytorch.

[37] Kaihua Tang, Yulei Niu, Jianqiang Huang, Jiaxin Shi, and Hanwang Zhang. 2020. Unbiased scene graph generation from biased training. In Proceedings of the IEEE/CVF Conference on Computer Vision and Pattern Recognition. 3716-3725.

[38] Kaihua Tang, Hanwang Zhang, Baoyuan Wu, Wenhan Luo, and Wei Liu. 2019. Learning to compose dynamic tree structures for visual contexts. In Proceedings of the IEEE Conference on Computer Vision and Pattern Recognition. 6619-6628.

[39] Damien Teney, Lingqiao Liu, and Anton van Den Hengel. 2017. Graph-structured representations for visual question answering. In Proceedings of the IEEE Conference on Computer Vision and Pattern Recognition. 1-9.

[40] Hongshuo Tian, Ning Xu, An-An Liu, and Yongdong Zhang. 2020. Part-Aware Interactive Learning for Scene Graph Generation. In Proceedings of the 28th ACM International Conference on Multimedia. 3155-3163.

[41] Tzu-Jui Julius Wang, Selen Pehlivan, and Jorma Laaksonen. 2020. Tackling the Unannotated: Scene Graph Generation with Bias-Reduced Models. In 31st British Machine Vision Conference 2020, BMVC 2020, Virtual Event, UK, September 7-10, 2020. BMVA Press.

[42] Wenbin Wang, Ruiping Wang, Shiguang Shan, and Xilin Chen. 2020. Sketching Image Gist: Human-Mimetic Hierarchical Scene Graph Generation. arXiv preprint arXiv:2007.08760 (2020).

[43] Meng Wei, Chun Yuan, Xiaoyu Yue, and Kuo Zhong. 2020. HOSE-Net: Higher Order Structure Embedded Network for Scene Graph Generation. In Proceedings of the 28th ACM International Conference on Multimedia (Seattle, WA, USA) (MM '20). Association for Computing Machinery, New York, NY, USA, 1846-1854.

[44] Danfei Xu, Yuke Zhu, Christopher B Choy, and Li Fei-Fei. 2017. Scene graph generation by iterative message passing. In Proceedings of the IEEE Conference on Computer Vision and Pattern Recognition. 5410-5419.

[45] Shaotian Yan, Chen Shen, Zhongming Jin, Jianqiang Huang, Rongxin Jiang, Yaowu Chen, and Xian-Sheng Hua. 2020. PCPL: Predicate-Correlation Perception Learning for Unbiased Scene Graph Generation. In Proceedings of the 28th ACM International Conference on Multimedia. 265-273.

[46] Jianwei Yang, Jiasen Lu, Stefan Lee, Dhruv Batra, and Devi Parikh. 2018. Graph r-cnn for scene graph generation. In Proceedings of the European Conference on Computer Vision. 670-685.

[47] Xu Yang, Kaihua Tang, Hanwang Zhang, and Jianfei Cai. 2019. Auto-encoding scene graphs for image captioning. In Proceedings of the IEEE Conference on Computer Vision and Pattern Recognition. 10685-10694.

[48] Ting Yao, Yingwei Pan, Yehao Li, and Tao Mei. 2018. Exploring visual relationship for image captioning. In Proceedings of the European Conference on Computer Vision. 684-699. 
[49] Fan Yu, Haonan Wang, Tongwei Ren, Jinhui Tang, and Gangshan Wu. 2020. Visual Relation of Interest Detection. In Proceedings of the 28th ACM International Conference on Multimedia (Seattle, WA, USA) (MM '20). Association for Computing Machinery, New York, NY, USA, 1386-1394.

[50] Cong Yuren, Hanno Ackermann, Wentong Liao, Michael Ying Yang, and Bodo Rosenhahn. 2020. NODIS: Neural Ordinary Differential Scene Understanding. CoRR abs/2001.04735 (2020).
[51] Alireza Zareian, Svebor Karaman, and Shih-Fu Chang. 2020. Bridging Knowledge Graphs to Generate Scene Graphs. arXiv preprint arXiv:2001.02314 (2020).

[52] Alireza Zareian, Haoxuan You, Zhecan Wang, and Shih-Fu Chang. 2020. Learning Visual Commonsense for Robust Scene Graph Generation. arXiv preprint arXiv:2006.09623 (2020).

[53] Rowan Zellers, Mark Yatskar, Sam Thomson, and Yejin Choi. 2018. Neural motifs: Scene graph parsing with global context. In Proceedings of the IEEE Conference on Computer Vision and Pattern Recognition. 5831-5840. 